\title{
Synthesis of polycyclic phosphonates via an intramolecular Diels-Alder reaction of 2-benzoylbenzalaldehyde and alkenyl phosphites
}

https://doi.org/10.1515/hc-2019-0011

Received November 1, 2018; accepted January 18, 2019.

\begin{abstract}
In this paper, we present a Lewis-acidpromoted reaction of 2-benzoylbenzaldehyde and trialkenyl phosphites, which resulted in the formation of polycyclic phosphonates. The reaction proceeded via nucleophilic attack of trialkenyl phosphite on the carbonyl carbon of 2-benzoylbenzaldehyde. The subsequent intramolecular Diels-Alder reaction led to the formation of the cyclic phosphonate.
\end{abstract}

Keywords: Intramolecular cycloaddition, Diels-Alder reaction, oxaphosphinane, isobenzofuran, cyclic phosphonate.

Cyclic phosphonates are often utilized as key intermediate reagents (synthetic intermediates) in the preparation of synthetically useful products and biologically active compounds [1,2]. Therefore, the synthesis of these compounds has attracted a great deal of research attention in the fields of synthetic organic, bioorganic, and medicinal chemistry [3-6]. Moreover, the development of new methods for the preparation of cyclic phosphonates has become very important in organic chemistry. For this purpose, the chemistry of isobenzofuran $[7,8,9]$ and the intramolecular Diels-Alder reaction $[10,11]$ are extremely interesting from a theoretical point of view. They represent a possible way to synthesize pharmaceutical candidate compounds, such as natural products and biologically active compounds with complicated structures in only a few short steps [3,12-20]. For example,

* Corresponding author: Kenji Yamana, Division of Liberal Arts and Sciences, Aichi Gakuin University, Araike 12, Iwasaki, Nisshin, Aichi 470-0195, Japan

Hirofumi Nakano, Department of Chemistry, Aichi University of Education, Igaya, Kariya, Aichi 448-8542, Japan the synthesis of alkaloid derivatives using Lewis acids has been reported by Yilin et al. [21,22]. Previously, we reported the formation of dialkyl isobenzofuran1-ylphosphonates by the reaction between $o$-phthalaldehyde and trialkyl phosphites in the presence of a Lewis acid (Scheme 1) [23,24].

In this study, we attempted to apply a Lewis-acidpromoted reaction of aromatic aldehydes and alkenyl phosphites to establish a new method for the one-step synthesis of polycyclic phosphonates.

Unfortunately, the reaction of $o$-phthalaldehyde 1 with triallyl phosphite $\mathbf{2 a}$ or tributenyl phosphite $\mathbf{2} \mathbf{b}$ failed to produce the intramolecular Diels-Alder adducts $\mathbf{4 a}$ and $\mathbf{4 b}$ (Scheme 2). We considered the possibility that isobenzofuran derivatives $\mathbf{3 a}$ and $\mathbf{3 b}$ were formed as intermediates, but decomposed due to their instability under these reaction conditions. Because of the low dienophilicity of the $\mathrm{C}=\mathrm{C}$ bond of allyl and butenyl groups, the intramolecular Diels-Alder reaction of $\mathbf{3}$ did not proceed smoothly. On the other hand, adducts $\mathbf{5 a}$ and $\mathbf{5 b}$ were formed from the reaction of 1 with trialkenyl phosphite and $N$-phenylmaleimide via intermediates $\mathbf{3} \mathbf{a}$ and $\mathbf{3 b}$. However, we considered the possibility to obtain the intramolecular adducts by stabilizing the intermediates $\mathbf{3} \mathbf{a}$ and $\mathbf{3 b}$.

Considering the previously reported stabilization effect of a phenyl group on the intermediate isobenzofuran-1-ylphosphonate [25], a similar approach was employed in this study. It was expected that the replacement of $\mathbf{1}$ by 2-benzoylbenzaldehyde $\mathbf{6}$ would stabilize the intermediates, isobenzofuran derivatives $7 \mathbf{a}$ and $\mathbf{7 b}$, due to a resonance effect derived from the phenyl group. The intramolecular adduct $\mathbf{9 b}$ (Scheme 3, 31\% yield) was generated from the intramolecular DielsAlder reaction of isobenzofuran derivative $\mathbf{7 b}$ and the subsequent aromatization of the product $\mathbf{8 b}$. However, these reaction conditions did not yield the intramolecular adduct 9a. This suggested that the strain of the 5 -membered ring in $\mathbf{8 a}$ is stronger than that of the 6-membered ring in $\mathbf{8 b}$.

¿ Open Access. (c) 2019 Kenji Yamana and Hirofumi Nakano, published by De Gruyter. @- 0 This work is licensed under the Creative Commons Attribution alone 4.0 License. 
74

K. Yamana and H. Nakano: Synthesis of polycyclic phosphonates via an intramolecular Diels-Alder reaction

DE GRUYTER
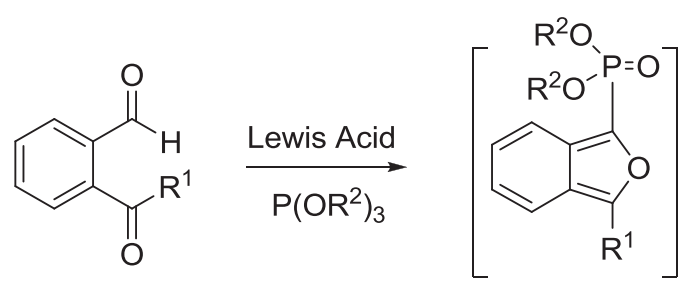

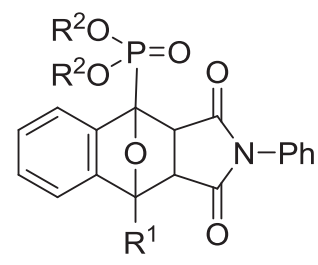

endow / exc

$$
\begin{aligned}
& \mathrm{R}^{1}=\mathrm{H}, \mathrm{Ph} \\
& \mathrm{R}^{2}=\mathrm{Me}, \mathrm{Et}, n-\mathrm{Pr}, i-\mathrm{Pr}
\end{aligned}
$$

Scheme 1<smiles>O=Cc1ccccc1C=O</smiles>

1

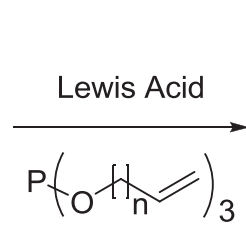

$2 a, 2 b$

a: $n=1$

b: $n=2$

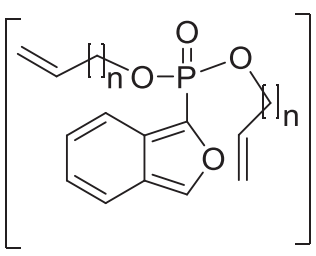

$3 a, 3 b$

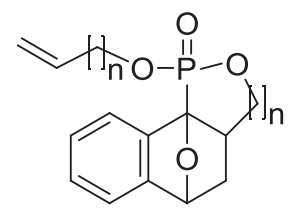

$4 a, 4 b$
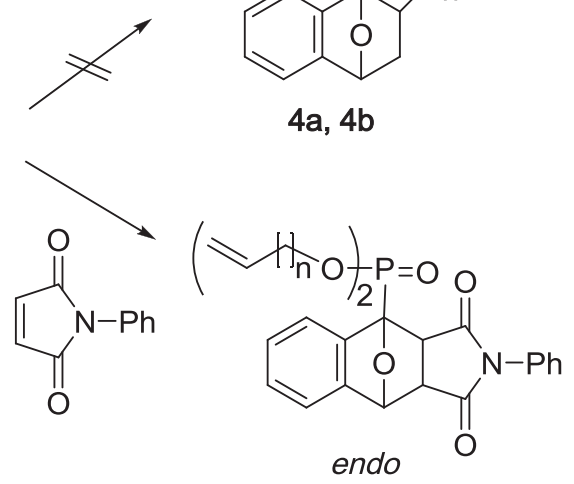

$5 a(47 \%), 5 b(27 \%)$

Scheme 2<smiles>O=Cc1ccccc1C(=O)c1ccccc1</smiles>

6

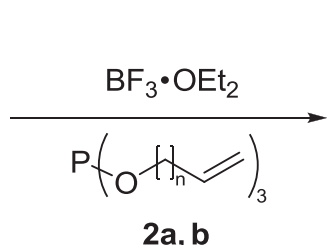

a: $n=1$

b: $n=2$

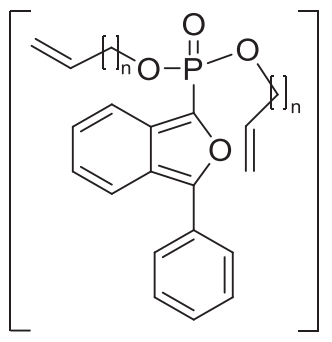

$7 a, b$

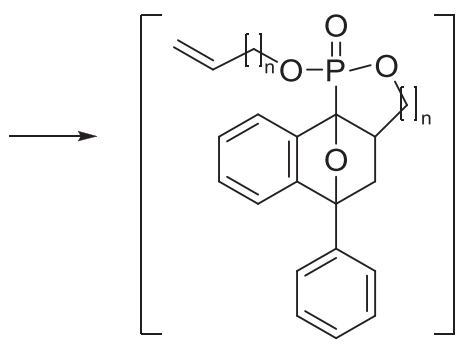

$8 a, b$
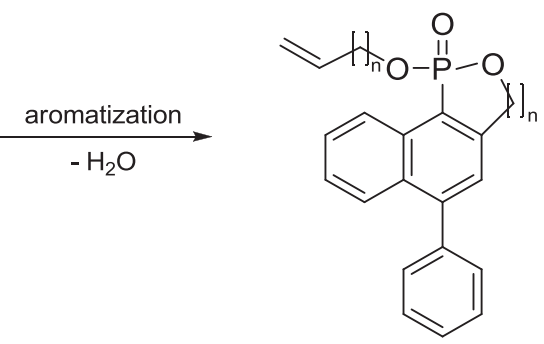

$9 a(0 \%), 9 b(31 \%)$

Scheme 3 
The fact that the yield of the intermolecular adduct 5a (47\%) from triallyl phosphite $\mathbf{2 a}$ was better than that of $\mathbf{5 b}(27 \%)$ from tributenyl phosphite $\mathbf{2 b}$ (Scheme 2) indicated that the reaction of $\mathbf{2 a}$ proceeded more smoothly than that of $\mathbf{2} \mathbf{b}$ because the allyl cation is more stable than the butenyl cation.

As previously reported, the rate limiting step in the formation of isobenzofuran-1-ylphosphonates is the alkyl group elimination from the trialkyl phosphite [25]. Moreover, the reaction proceeds more easily and the yield is increased when the alkyl group is smaller [Scheme 1, $\mathrm{R}^{1}=\mathrm{H}, \mathrm{R}^{2}=\mathrm{Me}(80 \%)$, Et (71\%), $\operatorname{Pr}(67 \%)$, and $i-\operatorname{Pr}(55 \%)$; endo/exo total yield). Similarly, the yield of polycyclic phosphonates should improve when the butenyl group of the trialkenyl phosphite is converted to a methyl group.

Therefore, dibutenyl methyl phosphite $\mathbf{2 c}$, where one of the butenyl groups is replaced by a methyl group, was employed in the reaction. This led to an increase in the total yield of the reaction to $67 \%$, with $9 \mathbf{b}(48 \%)$ being the major product after elimination of the methyl group (Scheme 4).

In summary, a new method for the synthesis of polycyclic phosphonates was developed, involving the treatment of 2-benzoylbenzaldehyde 6 with alkenyl phosphites, such as $\mathbf{2 b}$ and $\mathbf{2 c}$. Polycyclic phosphonates $\mathbf{9 b}$ and $\mathbf{9 c}$ were obtained via an intramolecular cycloaddition in only one step. These reactions provide a new approach to the generation of cyclic phosphonates.

\section{Experimental Details}

${ }^{1} \mathrm{H}$ NMR $(400 \mathrm{MHz})$ and ${ }^{13} \mathrm{C}$ NMR $(100 \mathrm{MHz})$ spectra were recorded in $\mathrm{CDCl}_{3}$ on a Bruker AVANCE III instrument. Tetramethylsilane was employed as an internal standard. The melting points were determined using a Yanako micro melting point apparatus and were uncorrected. Highresolution mass spectra were obtained using a JEOL JMST100GCV (EI) and microOTOF-QII (ESI).

\section{General Procedure for the Preparation of Intramolecular Diels-Alder Adducts}

$\mathrm{BF}_{3} \cdot \mathrm{OEt}_{2}(1 \mathrm{mmol})$ was added to a solution of 2-benzoylbenzaldehyde $6(1 \mathrm{mmol})$ in acetonitrile $(3 \mathrm{~mL})$ at<smiles>O=Cc1ccccc1C(=O)c1ccccc1</smiles><smiles>C/C=C\CCOP(C)OC</smiles><smiles>[R6]P(=O)(OCCC)c1oc(-c2ccccc2)c2ccccc12</smiles>

$7 b, 7 c$<smiles>[R6]P1(=O)OCC[C@H]2C[C@]3(c4ccccc4)O[C@]2(c2ccccc23)C1(C)C</smiles>

$8 b, 8 c$<smiles>[R20]P1CCCc2cc(-c3ccccc3)c3ccccc3c21</smiles>

$9 b(48 \%), 9 c(19 \%)$

$7-9 b: R=\xi($

7 - 9c: $R=M e$ 
Table 1 Yields of intramolecular Diels-Alder adducts $(9 \mathrm{a}-\mathrm{c})$ by the reaction of 2-benzoylbenzaldehyde (6) and phosphites (2a-c) ${ }^{\text {a }}$

\begin{tabular}{cccc}
\hline Entry & Phosphites & Adducts & Yields $^{\mathrm{b}}(\%)$ \\
\hline 1 & 2a & $9 \mathrm{a}$ & - \\
2 & 2b & $9 \mathrm{~b}$ & 31 \\
3 & 2c & $9 \mathrm{~b}, 9 \mathrm{c}$ & 48,19 \\
\hline
\end{tabular}

aReaction conditions: $\mathrm{BF}_{3} \cdot \mathrm{OEt}_{2}$ and 2-benzoylbenzaldehyde (1 equiv), $\mathrm{MeCN}, 0^{\circ} \mathrm{C}, 0.5 \mathrm{~h}$, followed by phosphites (1 equiv), $25^{\circ} \mathrm{C}, 48 \mathrm{~h}$. 'b ${ }^{\circ}$ solated.

$0{ }^{\circ} \mathrm{C}$. After stirring at this temperature for $0.5 \mathrm{~h}$, alkenyl phosphite $\mathbf{2} \mathbf{b}$ or $\mathbf{2 c}(1 \mathrm{mmol})$ was added and the mixture was stirred at $25^{\circ} \mathrm{C}$ for $48 \mathrm{~h}$. $\mathrm{HCl}$ solution was added to quench the reaction, and the organic layer was extracted with $\mathrm{CH}_{2} \mathrm{Cl}_{2}$, washed with $\mathrm{NaHCO}_{3}$, dried over anhydrous $\mathrm{Na}_{2} \mathrm{SO}_{4}$, and concentrated in vacuo. The residue was chromatographed on silica gel (AcOEt:Hexane $=1: 1)$ to give $\mathbf{9 b}$ or $\mathbf{9 c}$.

1-(But-3-en-1-yloxy)-6-phenyl-1H,3H,4H-1 $\lambda^{5}$-naphtho[1,2c][1,2] oxaphosphinin-1-one (9b).

The compound was obtained as a colorless oil; ${ }^{1} \mathrm{H}$ NMR $\left(400 \mathrm{MHz}, \mathrm{CDCl}_{3}\right): \delta 8.62(\mathrm{~d}, 1 \mathrm{H}, J=8.5 \mathrm{~Hz}), 7.87(\mathrm{~d}, 1 \mathrm{H}$, $J=8.5 \mathrm{~Hz}$ ), 7.62 (t, $1 \mathrm{H}, J=7.4 \mathrm{~Hz}), 7.43-7.52(\mathrm{~m}, 6 \mathrm{H}), 7.23$ (d, $1 \mathrm{H}, J=5.2 \mathrm{~Hz}$ ), 5.81 (ddt, $1 \mathrm{H}, J=17.1,10.3,6.8 \mathrm{~Hz}$ ), 5.13 (d, $1 \mathrm{H}, J=17.2 \mathrm{~Hz}), 5.08(\mathrm{~d}, 1 \mathrm{H}, J=10.2 \mathrm{~Hz}), 4.62-4.68(\mathrm{~m}$, $2 \mathrm{H}), 4.29-4.37$ (m, 1H), 4.23 (ddd, $1 \mathrm{H}, J=13.9,10.0,6.9 \mathrm{~Hz}$ ), 3.35-3.43 (m, 1H), 3.14 (ddd, $1 \mathrm{H}, J=17.2,7.3,4.0 \mathrm{~Hz}$ ), 2.48-2.53 (m, 2H); ${ }^{13} \mathrm{C}$ NMR (100 MHz, $\left.\mathrm{CDCl}_{3}\right): \delta 145.3(\mathrm{~d}$, $\left.{ }^{4} J_{\mathrm{C}, \mathrm{P}}=3.2 \mathrm{~Hz}\right), 142.0\left(\mathrm{~d},{ }^{2} J_{\mathrm{C}, \mathrm{P}}=6.3 \mathrm{~Hz}\right), 139.5,133.6,133.4(\mathrm{~d}$, $\left.{ }^{2} J_{\mathrm{C}, \mathrm{P}}=9.8 \mathrm{~Hz}\right), 130.9\left(\mathrm{~d},{ }^{3} \mathrm{C}_{\mathrm{C}, \mathrm{P}}=12.2 \mathrm{~Hz}\right), 129.7,128.4,128.0$, $127.8,127.2\left(\mathrm{~d},{ }^{3} J_{\mathrm{C}, \mathrm{P}}=14.9 \mathrm{~Hz}\right), 127.1\left(\mathrm{~d},{ }^{3} J_{\mathrm{C}, \mathrm{P}}=5.8 \mathrm{~Hz}, \mathrm{C}-5\right)$, 126.8 (C-7), 126.4 (C-8), 120.1 (d, $\left.{ }^{1} J_{C, P}=174.7 \mathrm{~Hz}, \mathrm{C}-10 \mathrm{a}\right), 117.7$ $\left(\mathrm{CH}_{2}=\mathrm{CH}-\right), 65.9\left(\mathrm{~d},{ }^{2} \mathrm{~J}_{\mathrm{C}, \mathrm{P}}=6.0 \mathrm{~Hz}, \mathrm{CH}_{2}\right), 65.3\left(\mathrm{~d},{ }^{2} \mathrm{~J}_{\mathrm{C}, \mathrm{P}}=7.0 \mathrm{~Hz}\right.$, $\left.\mathrm{CH}_{2}\right), 34.9\left(\mathrm{~d},{ }^{3} J_{\mathrm{C}, \mathrm{P}}=6.3 \mathrm{~Hz}, \mathrm{CH}_{2}\right), 32.3\left(\mathrm{~d},{ }^{3} J_{\mathrm{C}, \mathrm{P}}=7.1 \mathrm{~Hz}, \mathrm{C}-4\right)$. HRMS (EI) Calcd for $\mathrm{C}_{19} \mathrm{H}_{17} \mathrm{NO}_{3} \mathrm{P}\left(\mathrm{M}^{+}\right)$: 364.1228. Found: 364.1226.

\section{1-Methoxy-6-phenyl-1H,3H,4H-1 $\lambda^{5}$-naphtho[1,2-c][1,2] oxaphosphinin-1-one (9c).}

The compound was obtained as a colorless oil; ${ }^{1} \mathrm{H}$ NMR (400 MHz, $\left.\mathrm{CDCl}_{3}\right): \delta 8.61(\mathrm{~d}, 1 \mathrm{H}, J=8.5 \mathrm{~Hz}, \mathrm{ArH}), 7.87(\mathrm{~d}$, $1 \mathrm{H}, J=8.4 \mathrm{~Hz}, \mathrm{ArH}), 7.63$ (t, $1 \mathrm{H}, J=7.7 \mathrm{~Hz}, \mathrm{ArH}), 7.44-7.53$ (m, 6H, ArH), $7.24(\mathrm{~d}, J=5.2 \mathrm{~Hz}, 1 \mathrm{H}, \mathrm{ArH}), 4.62\left(\mathrm{~m}, 2 \mathrm{H}, \mathrm{CH}_{2}\right)$, $3.89\left(\mathrm{~d}, 3 \mathrm{H},{ }^{3} J_{\text {P.o-C.H }}=11.4 \mathrm{~Hz}, \mathrm{CH}_{3}\right), 3.34-3.41\left(\mathrm{~m}, 1 \mathrm{H}, \mathrm{CH}_{2}\right)$, 3.14-3.21(m, $\left.1 \mathrm{H}, \mathrm{CH}_{2}\right) ;{ }^{13} \mathrm{C}$ NMR (100 MHz, $\left.\mathrm{CDCl}_{3}\right): 8145.4(\mathrm{~d}$, $\left.{ }^{3} J_{\mathrm{C}-\mathrm{P}}=3.6 \mathrm{~Hz}, \operatorname{ArC}\right), 142.1\left(\mathrm{~d},{ }^{2} J_{\mathrm{C}-\mathrm{P}}=6.4 \mathrm{~Hz}, \operatorname{ArC}\right), 139.5(\operatorname{ArC})$, $133.4\left(\mathrm{~d},{ }^{2} J_{\mathrm{C}-\mathrm{P}}=9.9 \mathrm{~Hz}, \mathrm{ArC}\right), 130.9\left(\mathrm{~d},{ }^{3} J_{\mathrm{C}-\mathrm{P}}=12.1 \mathrm{~Hz}, \operatorname{ArC}\right)$,
129.7 (ArC), 128.4, 128.0, 127.8, $127.2\left(\mathrm{~d},{ }^{3} J_{\mathrm{C}-\mathrm{P}}=14.7 \mathrm{~Hz}, \mathrm{ArC}\right)$, 126.9 (d, ${ }^{3} J_{C-P}=5.7 \mathrm{~Hz}, \operatorname{ArC}$ ), 126.8 (ArC), 126.4, 120.0 $\left(\mathrm{d}, J_{\text {C.P }}=174.1 \mathrm{~Hz}, \operatorname{ArC}-\mathrm{P}\right), 66.0\left(\mathrm{~d},{ }^{2} J_{\text {C-O-P }}=5.9 \mathrm{~Hz}, \mathrm{CH}_{2}\right), 52.7$ (d, $\left.{ }^{3} \mathrm{C}_{\mathrm{C}-\mathrm{O}-\mathrm{P}}=6.8 \mathrm{~Hz}, \mathrm{CH}_{2}\right), 32.3\left(\mathrm{~d},{ }^{3} \mathrm{~J}_{\mathrm{C}-\mathrm{O}-\mathrm{P}}=7.1 \mathrm{~Hz}, \mathrm{CH}_{2}\right.$ ). HRMS (EI) Calcd for $\mathrm{C}_{19} \mathrm{H}_{17} \mathrm{NO}_{3} \mathrm{P}\left(\mathrm{M}^{+}\right)$: 324.0915. Found: 324.0907.

\section{Bis(but-3-enyl) methyl phosphite (2c).}

The compound was obtained as a colorless oil; ${ }^{1} \mathrm{H}$ NMR $\left(400 \mathrm{MHz}, \mathrm{CDCl}_{3}\right): \delta$ 5.76-5.85 (m, 2H), 5.06-5.14 (m, 4H), $3.51(\mathrm{dt}, 4 \mathrm{H}, J=7.8,6.9 \mathrm{~Hz}), 3.51(\mathrm{~d}, 3 \mathrm{H}, J=10.4 \mathrm{~Hz}$ ), 2.36-2.45 (m, 4H); ${ }^{13} \mathrm{C}$ NMR (100 MHz, $\left.\mathrm{CDCl}_{3}\right): \delta 134.5$, 117.7, $61.7\left(\mathrm{~d},{ }^{2} \mathrm{~J}_{\mathrm{C}, \mathrm{P}}=11.7 \mathrm{~Hz}\right), 49.0\left(\mathrm{~d},{ }^{2} J_{\mathrm{C}, \mathrm{P}}=9.3 \mathrm{~Hz}\right), 35.6\left(\mathrm{~d},{ }^{4} \mathrm{~J}_{\mathrm{C}, \mathrm{P}}=\right.$ $5.0 \mathrm{~Hz}$ ). HRMS (ESI Negative) Calcd for $\mathrm{C}_{19} \mathrm{H}_{17} \mathrm{NO}_{3} \mathrm{P}(\mathrm{M}+\mathrm{H})$ : 205.0994. Found: 205.0637.

\section{Bis(prop-2-enyl) [(3a $\left.R^{\star}, 4 S^{\star}, 9 S^{\star}, 9 \mathrm{a} R^{\star}\right)$-1,3-dioxo- 2-phenyl-1,2,3,3a,9,9a-hexahydro-4H-4,9-epoxybenzo[f] isoindol-4-yl]phosphonate (endo) (5a).}

The compound was obtained as a colorless oil; ${ }^{1} \mathrm{H}$ NMR (400 MHz, $\left.\mathrm{CDCl}_{3}\right): \delta$ 7.52-7.61 (m, 1H), 7.34-7.40 (m, 3H), 7.25-7.28 (m, 3H), 6.38-6.42 (m, 2H), 6.02-6.11 (m, 1H), 5.87-5.96 (m, 2H), 5.44-5.49 (m, 1H), 5.28-5.35 (m, 2H), 5.20-5.22 (m, 1H), 4.87-4.98 (m, 2H), 4.64-4.76 (m, $2 \mathrm{H})$, $4.27(\mathrm{dd}, 1 \mathrm{H}, J=9.0,8.6 \mathrm{~Hz}), 4.07(\mathrm{dd}, 1 \mathrm{H}, J=8.5,5.8 \mathrm{~Hz})$; ${ }^{13} \mathrm{C}$ NMR $\left(67.80 \mathrm{MHz}, \mathrm{CDCl}_{3}\right): \delta 172.6,171.5,140.7,140.7,139.7$, 139.6, 132.8, 132.7, 132.4, 132.4, 130.8, 129.0, 128.9, 128.7, $128.6,126.3,125.8,122.1,121.3,118.7,118.5,86.2\left(\mathrm{~d}, J_{\mathrm{C}, \mathrm{P}}=\right.$ $191.3 \mathrm{~Hz}), 81.6\left(\mathrm{~d},{ }^{3} \mathrm{C}_{\mathrm{C}, \mathrm{P}}=15.4 \mathrm{~Hz}\right), 68.5\left(\mathrm{~d},{ }^{2} \mathrm{~J}_{\mathrm{C}, \mathrm{P}}=6.0 \mathrm{~Hz}\right)$, $68.0\left(\mathrm{~d},{ }^{2} J_{\mathrm{C}, \mathrm{P}}=6.2 \mathrm{~Hz}\right), 50.7\left(\mathrm{~d},{ }^{3} J_{\mathrm{C}, \mathrm{P}}=6.6 \mathrm{~Hz}\right), 49.7\left(\mathrm{~d},{ }^{3} J_{\mathrm{C}, \mathrm{P}}=\right.$ $4.4 \mathrm{~Hz}$ ). HRMS (ESI Negative) Calcd for $\mathrm{C}_{24} \mathrm{H}_{21} \mathrm{NO}_{6} \mathrm{P}\left(\mathrm{M}-\mathrm{H}^{+}\right)$: 450.1106. Found: 450.1169.

\section{Bis(but-3-enyl) [(3a $\left.R^{\star}, 4 S^{\star}, 9 S^{\star}, 9 \mathrm{a} R^{\star}\right)$-1,3-dioxo-2-phe- nyl-1,2,3,3a,9,9a-hexahydro-4H-4,9-epoxybenzo[f] isoindol-4-yl]phosphonate (endo) (5b).}

The compound was obtained as a colorless oil; ${ }^{1} \mathrm{H}$ NMR (400 MHz, $\left.\mathrm{CDCl}_{3}\right)$ : $\delta$ 7.55-7.58 (m, 1H), 7.33-7.39 (m, 3H), 7.24-7.26 (m, 3H), 6.38-6.41 (m, 2H), 5.81-5.91 (m, 2H), 5.66-5.76 (m, 1H), 5.01-5.20 (m, 4H), 4.47 (dt, $2 \mathrm{H}, J=6.8$, $8.0 \mathrm{~Hz}, 2 \mathrm{H}), 4.18-4.29$ (m, 3H), $4.05(\mathrm{dd}, 1 \mathrm{H}, J=5.8,5.8 \mathrm{~Hz}$ ), 2.56-2.61 (m, 2H), $2.41(\mathrm{dt}, 2 \mathrm{H}, J=6.7,6.7 \mathrm{~Hz}) ;{ }^{13} \mathrm{C} \mathrm{NMR}$ $\left(67.80 \mathrm{MHz}, \mathrm{CDCl}_{3}\right): \delta 172.6,171.5,140.8,140.7,139.8,139.8$, $133.5,133.2,130.8,129.1,129.0,128.8,128.7,128.5,126.3$, 122.1, 121.2, 117.9, 117.7, $86.2\left(\mathrm{~d}, J_{\mathrm{C}, \mathrm{P}}=191.1 \mathrm{~Hz}\right), 81.6\left(\mathrm{~d},{ }^{3} J_{\mathrm{C}, \mathrm{P}}=\right.$ $15.3 \mathrm{~Hz}), 67.0\left(\mathrm{~d},{ }^{2} J_{\mathrm{C}, \mathrm{P}}=6.5 \mathrm{~Hz}\right), 66.7\left(\mathrm{~d},{ }^{2} J_{\mathrm{C}, \mathrm{P}}=6.5 \mathrm{~Hz}\right), 50.7$ $\left(\mathrm{d},{ }^{3} J_{\mathrm{C}, \mathrm{P}}=6.6 \mathrm{~Hz}\right), 49.7\left(\mathrm{~d},{ }^{3} J_{\mathrm{C}, \mathrm{P}}=4.3 \mathrm{~Hz}\right), 35.1\left(\mathrm{~d},{ }^{3} J_{\mathrm{C}-\mathrm{P}}=\right.$ $5.5 \mathrm{~Hz}), 34.8\left(\mathrm{~d},{ }^{3} J_{\mathrm{C}, \mathrm{P}}=5.8 \mathrm{~Hz}\right.$ ). HRMS (ESI Negative) Calcd for $\mathrm{C}_{26} \mathrm{H}_{25} \mathrm{NO}_{6} \mathrm{P}\left(\mathrm{M}-\mathrm{H}^{+}\right)$: 478.1419. Found: 478.1476. 
Acknowledgements: We would like to thank Editage (www. editage.jp) for English language editing.

\section{References}

[1] Zhang, H.; Tsukuhara, R.; Tigyi, G.; Prestwich, G. D. Synthesis of cyclic phosphonate analogues of (lyso)phosphatidic acid using a ring-closing metathesis reaction. J. Org. Chem. 2006, 71, 6061-6066.

[2] Stoianova, D. S.; Whitehead, A.; Hanson, P. R. P-Heterocyclic building blocks: Application to the stereoselective synthesis of P-sugars. J. Org. Chem. 2005, 70, 5880-5889.

[3] Unoh, Y.; Hashimoto, Y.; Takeda, D.; Hirano, K.; Satoh, T.; Miura, M. Rhodium(III)-catalyzed oxidative coupling through $\mathrm{C}-\mathrm{H}$ bond cleavage directed by phosphinoxy groups. Org. Lett. 2013, 15, 3258-3261.

[4] López-Cortina, S.; Basiulis, D. I.; Marsi, K. L.; MuñozHernández, M. A.; Ordoñez, M.; Fernández-Zertuche, M. Synthesis of new 1,3-oxaphosphorinanium salts. Stereochemistry of hydroxide-induced displacement of methoxide ion. J. Org. Chem. 2005, 70, 7473-7478.

[5] Li, Z.; Han, J.; Jiang, Y.; Browne, P.; Knox, R. J.; Hu, L. Nitrobenzocyclophosphamides as potential prodrugs for bioreductive activation: synthesis, stability, enzymatic reduction, and antiproliferative activity in cell culture. Bioorg. Med. Chem. 2003, 11, 4171-4178.

[6] Pirat, J.-L.; Monbrun, J.; Virieux, D.; Volle, J.-N.; Tillard, M.; Cristau, H.-J. Diastereoselective addition of $2 \mathrm{H}$-2-oxo-1,4,2oxazaphosphinanes to aldehydes and imines. J. Org. Chem. 2005, 70, 7035-7041.

[7] Haddadin, M. J. Isobenzofuran. Heterocycles. 1978, 9, 865-901.

[8] Haddadin, M. J.; Agha, B. J.; Tabri, R. F. Syntheses of some furans and naphtho[2,3-c] derivatives of furan, pyrrole, and thiophene. J. Org. Chem. 1979, 44, 494-497.

[9] Ohmura, H.; Mikami, K. Novel isobenzofuran generation from silylated lactol leading to desilylated or silylated adducts depending on the choice of metal fluorides. Synlett. 2002, 1868-1870.

[10] Ohba, M.; Izuta, R. Effect of copper(II) triflate on intramolecular Diels-Alder reaction of oxazole-olefins. Heterocycles. 2001, 55, 823-826.

[11] Padwa, A.; Eidell, C. K.; Lynch, S. M. A new construct of the cis-3a-aryloctahydroindole skeleton via the [4+2] cycloaddition of furanyl carbamates. Heterocycles. 2002, 58, 227-242.
[12] Zhang, L.; Koreeda, M. Radical deoxygenation of hydroxyl groups via phosphites. J. Am. Chem. Soc. 2004, 126, 13190-13191.

[13] Peng, A.-Y.; Ding, Y.-X. Synthesis of $2 H-1,2$-oxaphosphorin 2-oxides via $\mathrm{Ag}_{2} \mathrm{CO}_{3}$-catalyzed cyclization of (Z)-2-alken-4ynylphosphonic monoesters. Org. Lett. 2005, 7, 3299-3301.

[14] Mo, J.; Kang, D.; Eom, D.; Kim, S. H.; Lee, P. H. Gold-catalyzed sequential alkyne activation for the synthesis of 4,6-disubstituted phosphorus 2-pyrones. Org. Lett. 2013, 15, 26-29.

[15] Li, B.; Zhou, B.; Lu, H.; Ma, L.; Peng, A.-Y. Phosphaisocoumarins as a new class of potent inhibitors for pancreatic cholesterol esterase. Eur. J. Med. Chem. 2010, 45, 1955-1963.

[16] Li, X.; Zhang, D.; Pang, H.; Shen, F.; Fu, H.; Jiang, Y.; Zhao, Y. Synthesis of a diverse series of phosphacoumarins with biological activity. Org. Lett. 2005, 7, 4919-4922.

[17] Peng, A.-Y.; Ding, Y.-X. Synthesis of phosphaisocoumarins via iodocyclization. Org. Lett. 2004, 6, 1119-1121.

[18] Seo, J.; Park, Y.; Jeon, I.; Ryu, T.; Park, S.; Lee, P. H. Synthesis of phosphaisocoumarins through rhodium-catalyzed cyclization using alkynes and arylphosphonic acid monoesters. Org. Lett. 2013, 15, 3358-3361.

[19] Park, Y.; Jeon, I.; Shin, S.; Min, J.; Lee, P. H. Rutheniumcatalyzed $\mathrm{C}-\mathrm{H}$ activation/cyclization for the synthesis of phosphaisocoumarins. J. Org. Chem. 2013, 78, 10209-10220.

[20] Jeon, W. H.; Son, J.-Y.; Kim, S.-E.; Lee, P. H. Phosphaannulation of aryl- and benzylphosphonic acids with unactivated alkenes via palladium-catalyzed $\mathrm{C}-\mathrm{H}$ activation/oxidative cyclization reaction. Adv. Synth. Catal. 2015, 357, 811-817.

[21] Zhang, Y.; Hubbard, J. W.; Akhmedov, N. G.; Petersen, J. L.; Söderberg, B. C. G. Total synthesis of the tetracyclic indole alkaloid Ht-13-B. J. Org. Chem. 2015, 80, 4783-4790.

[22] Zhang, Y.; McArdle, I. W.; Hubbard, J. W.; Akhmedov, N. G.; Söderberg, B. C. G. Total synthesis of the tetracyclic indole alkaloid Ht-13-A. Tetrahedron Lett. 2016, 57, 2865-2867.

[23] Yamana, K.; Nakano, H. The Lewis acid-promoted reactions of $o$-phthaladehyde with trialkyl phosphites: Formation of 1-dialkoxyphosphorylisobenzofuran. Tetrahedron Lett. 1996, 37, 5963-5966.

[24] Yamana, K.; Ibata, T.; Nakano, H. The formation of dialkyl isobenzofuran-1-ylphosphonates by Lewis acid promoted reaction of $o$-phthalaldehyde with trialkyl phosphites. Synthesis. 2006, 4124-4130. 